\title{
EFFECTS OF DROUGHT STRESS ON ENDOGENOUS HORMONES AND OSMOTIC REGULATORY SUBSTANCES OF COMMON BEAN (PHASEOLUS VULGARIS L.) AT SEEDLING STAGE
}

\author{
WANG, Q. ${ }^{1,2}-$ LIN $_{\text {F }} .^{1 *}-$ WeI, S. H. ${ }^{2}-$ MENG, X. X. ${ }^{2}-$ YIN, Z. G. ${ }^{2}-$ GUO, Y. F. ${ }^{2}-$ YANG, G. D. ${ }^{3}$ \\ ${ }^{1}$ College of Bioscience and Biotechnology, Shenyang Agricultural University \\ No. 120 Dongling Road, Shenyang, 110866 Liaoning, China \\ ${ }^{2}$ Crop Resources Institute of Heilongjiang Academy of Agricultural Sciences \\ 150086 Heilongjiang, China \\ ${ }^{3}$ Keshan Branch of Heilongjiang Academy of Agricultural Sciences \\ 161600 Heilongjiang, China \\ *Corresponding author \\ e-mail:fenglinsn@126.com \\ (Received 21 ${ }^{\text {st }}$ Jan 2019; accepted $20^{\text {th }}$ Feb 2019)
}

\begin{abstract}
Drought is an important factor that restricts the growth of common bean. In the present study, Longyundou 10 (non-drought-resistant variety) and Longyundou 17 (drought-resistant variety) were selected to analyze the effects of drought stress on endogenous hormones and osmotic regulatory substances of common bean at seedling stage. Under mild drought stress, the ABA accumulation and response of drought-resistant Longyundou 17 were stronger than that of no drought-resistant Longyundou 10. The effects of CTK and IAA on drought resistance of common bean at seedling stage are complicated. Under normal water condition, the soluble protein content of Longyundou 10 was slightly higher than that of Longyundou 17. However, there was no significant difference in the degree of inhibition between Longyundou 10 and 17. Therefore, we believe that the soluble protein of common bean has little effect on resisting the threat of drought stress. The content of proline and soluble sugar in common bean responded strongly to drought stress, these two osmotic regulatory substances in drought-resistant varieties were higher than those in drought-resistant varieties. Therefore, ABA, proline and soluble sugar content can be used as important indicators for breeding of drought-resistant Varieties of common bean at seedling stage. Keywords: common bean, drought stress, ABA, proline, soluble sugar
\end{abstract}

\section{Introduction}

Common bean (Phaseolus vulgaris L.) is the most popular edible bean widely cultivated in more than 90 countries and regions around the world (Acostagallegos et al., 2007). Common bean is rich in protein, unsaturated fatty acid, dietary fiber and other nutrients (Kuto et al., 2003; Yang et al., 2016). Drought stress is not only a hot research topic, but also a common adversity for crop growth. It is also a decisively restrictive factor for sustainable agricultural development which always threatens food security (Schindler et al., 2007). With the change of global climate, the drought situation in common bean producing areas is getting worse and worse. According to statistics, $60 \%$ of common bean producing areas in the world suffer from drought, and the yield reduction in Kenya and South Africa is as high as $80 \%$ (Beebe et al., 2008; Franca et al., 2000).

Under drought conditions, it is a complex physiological change for plants to respond to adversity. Many researches reported that drought causes responses of endogenous 
hormones in plants, which regulate and control plant physiological activities to adapt to drought stress (Yordanov et al., 2000; Sauter et al., 2001; Farooq et al., 2009). Abscisic acid (ABA), indole acetic acid (IAA) and cytokinin (CTK) are drought stress response hormones (Bartels et al., 2005; Acharyaet a., 2009; Tang et al., 2005), which can regulate plant physiological and biochemical processes and enhance drought resistance of crops. ABA exists in almost all higher plants and participates in many physiological processes during plant growth and development, such as stomatal movement, fruit maturation, seed dormancy and germination (Cutler et al., 2010). Under drought stress, ABA concentration in plants increased, which promote stoma to close to reduce transpiration loss, thereby to enhance plant drought resistance (Schroeder et al., 2001; Verslues et al., 2005).

IAA is a kind of plant endogenous hormones containing an unsaturated aromatic ring and an acetic acid side chain. Most IAA is concentrated in tissues and organs with strong division and growth metabolism, such as in root tips, young leaves, developing seeds and fruits. Drought stress can affect the change of IAA concentration. The mechanism of IAA drought regulation is complex. CTK is a kind of adenine derivative. Under drought stress, the regulation mechanism of CTK varies in drought degrees and crops. Osmotic regulation is the physiological mechanism which plants are adapted to water stress. Under water stress, plant cells can actively change the content of osmotic regulatory substances of small molecules or their existing state in plants to regulate osmotic potential in vacuoles and ensure normal growth and development of plants. Soluble sugars, proline and soluble proteins are considered to be important osmotic regulatory substances in plants, which can respond positively to drought stress. It has been reported that under drought stress, the proline content of wheat leaves increased and the soluble protein content decreased (Bai et al., 2007). Proline and soluble sugar are the main osmotic regulatory substances of common bean, which can maintain the leaf water balance (Li et al., 2014). Under drought stress, the content of osmotic regulatory substances in common bean was different among different drought-resistant varieties (Rosales et al., 2012; Lizana et al., 2006).

Drought is the second major factor restricting the production of common bean besides diseases (Schneider et al., 1997). Therefore, drought resistance of common bean has become one of the important indexes in breeding and production ( $\mathrm{Li}$ et al., 2014; Cortés et al., 2012). In the present study, the changes of endogenous hormones and osmotic regulatory substances in common bean were studied in order to provide technical guidance and theoretical support for efficient production and breeding of drought-resistant of common bean varieties.

\section{Materials and methods}

Both Longyundou 10 (non-drought-resistant variety) and Longyundou 17 (droughtresistant variety) were provided by Crop Breeding Research Institute of Heilongjiang Academy of Agricultural Sciences, Heilongjiang province, China.

The experiment was carried out in an artificial climate chamber with humidity of $60 \%+10 \%, 27{ }^{\circ} \mathrm{C}$ under 16 -h light conditions, $16^{\circ} \mathrm{C}$ under 8 -h dark conditions. The experimental plots were completely randomized with three replications and three water treatments at each site. Mild drought stress (LS): Relative water content of soil $(\mathrm{RWC})=60 \%+5 \%$; severe drought stress $(\mathrm{SS}): \mathrm{RWC}=45 \%+5 \%$; control: $\mathrm{RWC}=75 \%+5 \%$ under normal water condition. Seeds were planted in plastic pots 
filled with a potting mixture of 3 perlite : 3 vermiculite : 4 nursery soil. Each plastic pot contained $2 \mathrm{~kg}$ potting mixture and 3 seeds. From sowing to the appearance of the second true leaf, the soil was controlled under normal water condition ( $\mathrm{RWC}=75 \%+5 \%)$. When the second trifoliate leaves are fully developed and mature, the soil moisture content of each treatment was monitored and controlled to reach the set target by weighing method every morning and evening.

On the 2nd, 4th, 6th, 8th, 10th, 12nd, 14th and 16th days after drought stress treatment, the parietal lobe of the second mature trifoliate were taken from 9:00 to 9:30 a.m. The contents of ABA, CTK, IAA, free proline, soluble sugar and soluble protein were determined by ELISA. All the kits are provided by Shanghai Enzyme-linked Biotechnology Co., Ltd. Microsoft Excel 2010 and SPSS 18.0 software are employed for data collation and analysis.

\section{Results}

\section{Effects of drought stress on ABA content of common bean}

$\mathrm{ABA}$ is a stress response hormone, which can accumulate rapidly when stress occurs and play a significant role in plant response to stress. As shown in Figure 1, at the beginning of severe drought stress, ABA content of Longyundou 17 increased abnormally. In addition, the accumulation of ABA content in common bean leaves showed a single-peak curve at seedling stage. The peaks of ABA content of Longyundou 17 in mild drought stress, severe drought stress and control treatments were $941.81 \mathrm{ng} \mathrm{g}^{-1}, 829.97 \mathrm{ng} \mathrm{g}^{-1}$ and $747.16 \mathrm{ng} \mathrm{g}^{-1}$, respectively. The peaks of ABA content of Longyundou 10 in mild drought stress, severe drought stress and control treatments were $849.33 \mathrm{ng} \mathrm{g}^{-1}, 731.04 \mathrm{ng} \mathrm{g}^{-1}$ and $724.58 \mathrm{ng} \mathrm{g}^{-1}$, respectively.
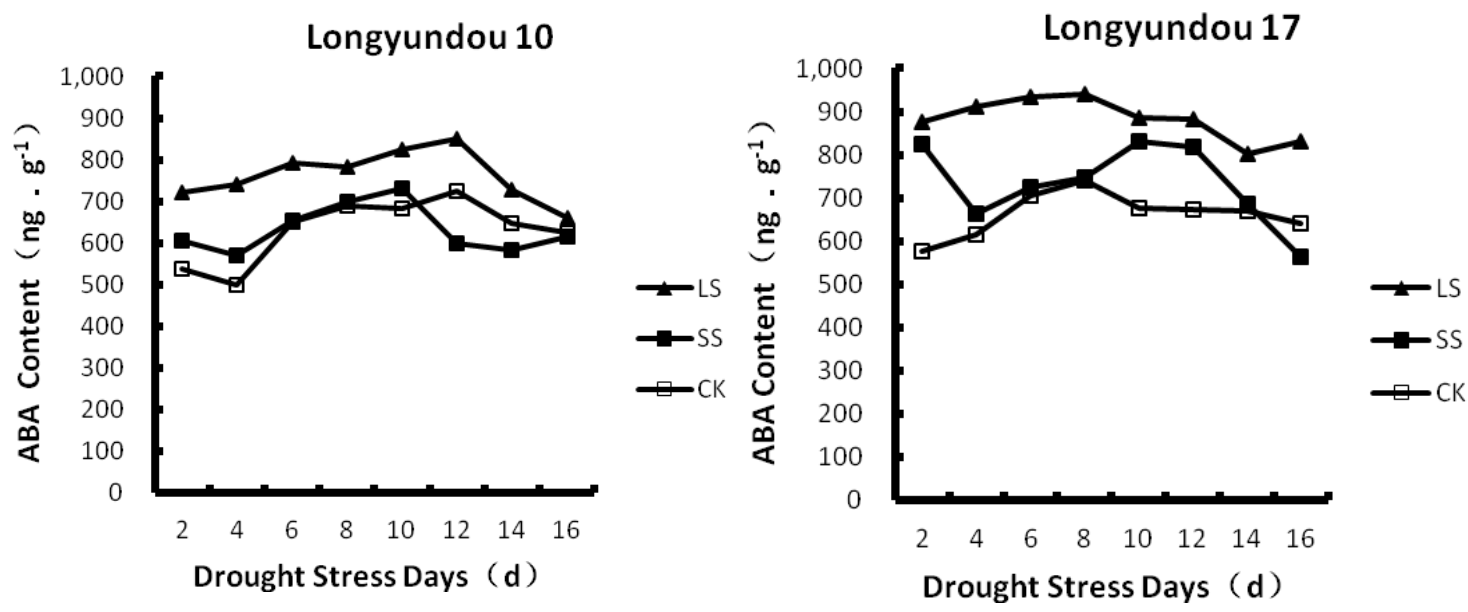

Figure 1. Changes of ABA content in common bean leaves under drought stress

Under mild drought stress, the accumulation of ABA in leaves increased significantly, but with the increase of water stress time, the content of ABA in severe drought stress began to decrease and accumulated slowly, which indicated that mild drought stress stimulated the ABA response. Common bean could alleviate the effect of water stress through the accumulation of $\mathrm{ABA}$ in a certain period of time, but severe drought stress can destroy the formation and accumulation of ABA. 
ABA content in leaves of drought-resistant variety Longyundou 17 was slightly higher than that of Longyundou 10. Under drought stress, the increase of ABA content was also greater than that of common bean varieties with weak drought resistance. On the 12th day after severe drought treatment, ABA content of Longyundou 10 was lower than that of normal water treatment, while Longyundou 17 decreased on the 16th day after severe drought treatment, indicating that the accumulation and response of ABA under drought stress were stronger than those of non-drought resistant varieties.

\section{Effect of drought stress on CTK content of common bean}

As shown in Figure 2, under normal water condition, CTK content fluctuated in the leaves of common bean at seedling stage, but there is no distinct tendency. Under drought stress, CTK content increased first, then decreased, and then increased again. The performance of CTK content was different among different varieties, which indicated that the role of CTK in drought resistance of common bean at seedling stage is complex.

There were no significant differences in CTK content of Longyundou 10 among drought stress treatments, but the change tendency was slightly different. When Longyundou 10 was treated with severe drought stress for 2-4 days, the content of CTK content increased significantly, with an average increase of $50.6 \%$ compared with the control. After 6 days of treatment, CTK content declined sharply, slightly lower than the control treatment on the 8th day, and increased again after 12 days of treatment. Under mild drought stress, the CTK content in Longyundou 10 was significantly higher than that under normal water condition after 2nd-6th and 16th days of treatment, but increased slightly after other treatments, even decreased slightly on the 8th day of treatment.

Except for the 4th day after treatment, the CTK content of Longyundou 17 under severe stress treatment was higher than that in mild stress treatment. The CTK content under drought stress was higher than that under no stress treatment, indicating that drought stress stimulated the CTK production in Longyundou 17.
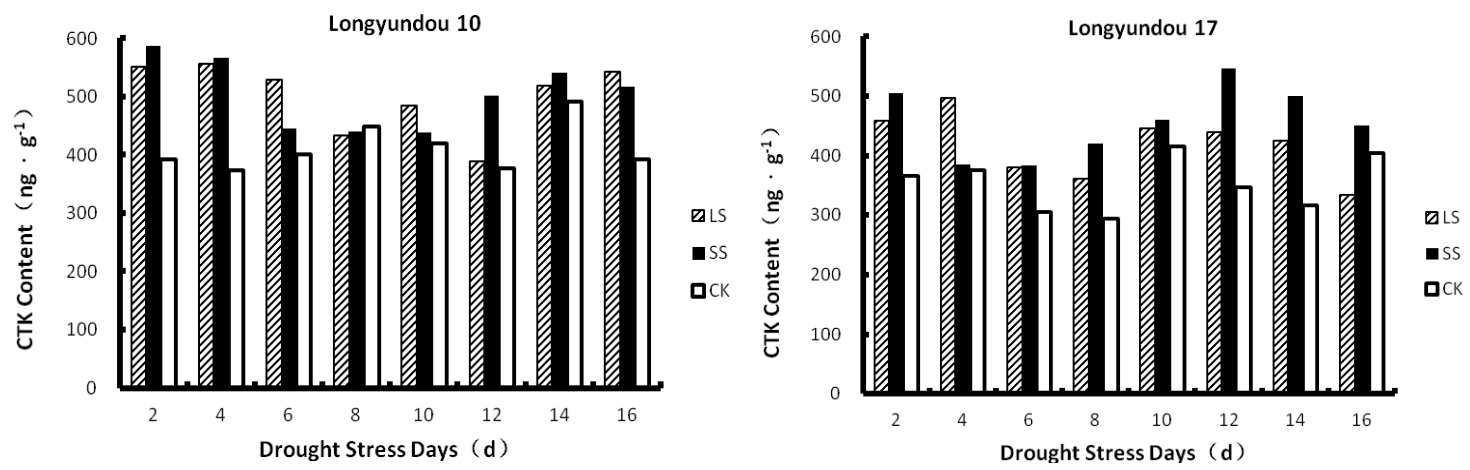

Figure 2. Changes of CTK content in common bean leaves under drought stress

\section{Effect of drought stress on IAA content of common bean}

As shown in Figure 3, IAA content of common bean showed a downward trend of fluctuations under drought stress. The change of IAA content under drought stress was more complex. The changes of IAA content under both severe and mild drought stress were alternate, and no drought stress showed greater impact on IAA content. At the initial stage 
of drought stress, IAA content was higher than that under no stress. However, with the prolongation of drought stress time, IAA accumulation was inhibited and content began to decline. Especially from the 8th day after drought treatment, IAA content of Longyundou 10 was lower than that of control. The IAA content of Longyundou 17 was slightly higher than Longyundou 10 in all treatments.
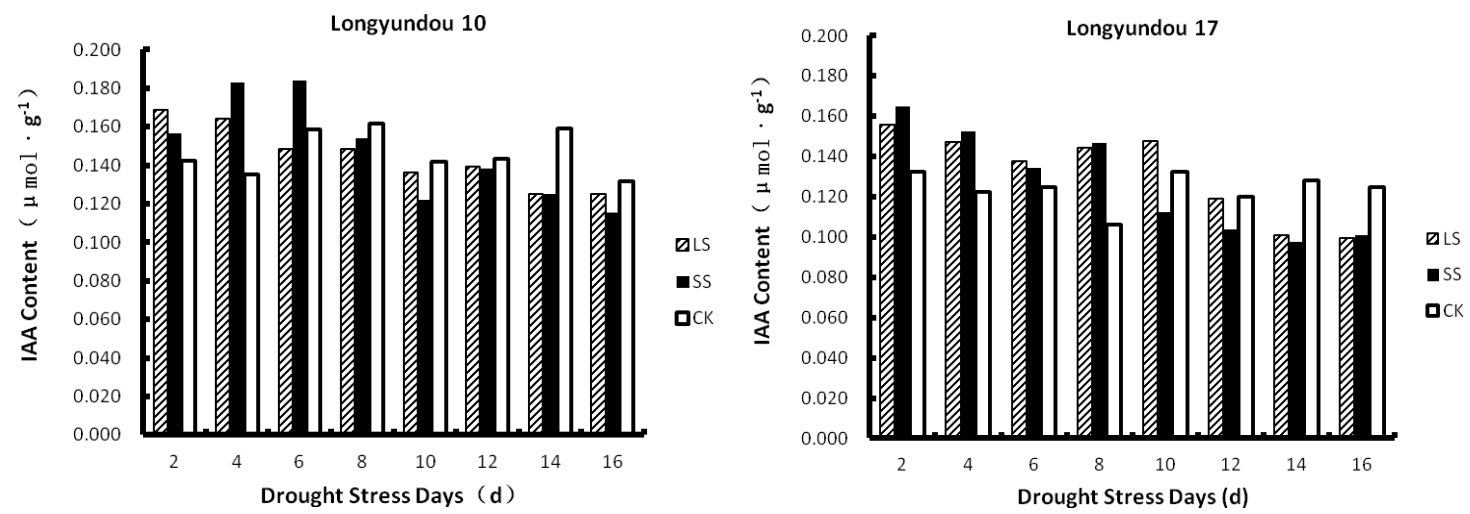

Figure 3. Changes of IAA content in common bean leaves under drought stress

\section{Effects of drought stress on free proline content of common bean}

In Figure 4, under different degrees of drought stress, the free proline content in common bean leaves increased significantly with the maximum increase more than 1.6 times than that under non-drought stress, while free proline accumulation fluctuated under mild drought stress.
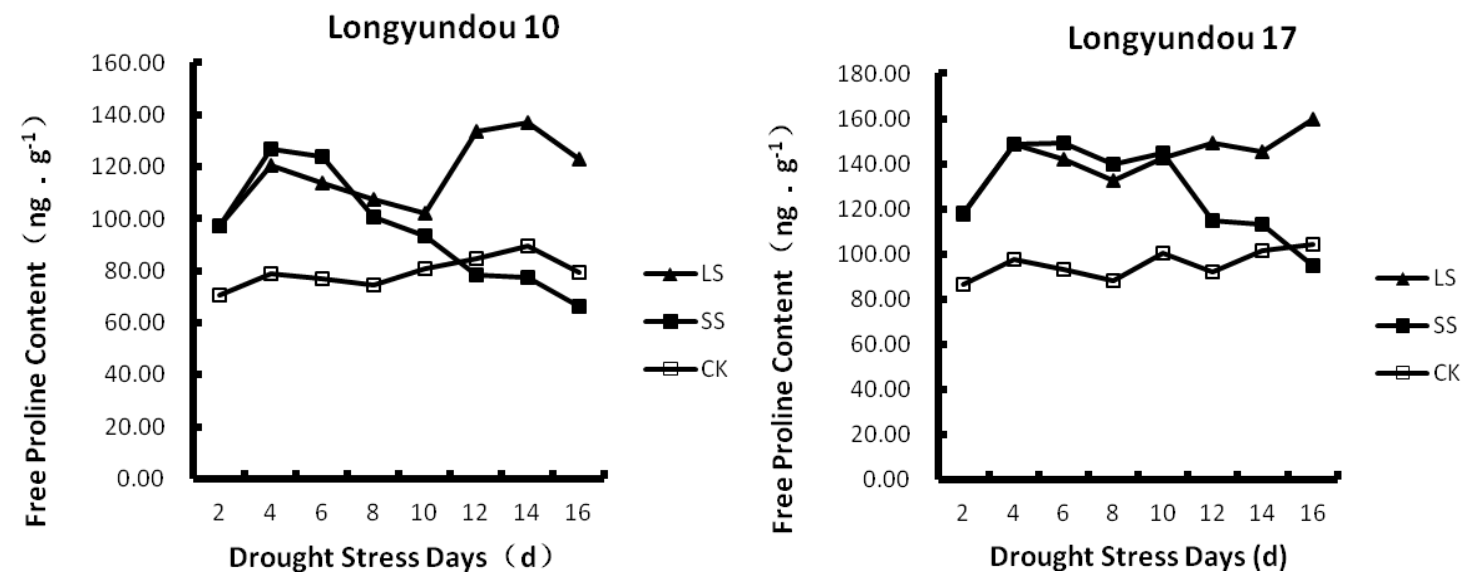

Figure 4. Changes of free proline content in common bean leaves under drought stress

Under severe drought stress, free proline content tends to increase at first and then decrease. At the beginning of drought treatment, the content of proline under severe drought stress was higher than that under other water treatments. With the prolongation of drought stress time, the accumulation of proline under severe drought stress was inhibited and the content of proline decreased. Longyundou 17 maintained high free proline accumulation within 10 days under severe drought stress, but began to decrease 12 days after treatment, which was lower than that under mild drought stress. The free proline content of Longyundou 10 under 
mild stress was higher than that under severe stress after 8 days of drought treatment, which indicated that Longyundou 17 could maintain higher free proline accumulation for a long time under severe stress than Longyundou 10, and its osmotic regulation ability was also higher than Longyundou 10. The free proline content of Longyundou 17 was higher than Longyundou 10 in all treatments, and the increase of free proline under drought stress was also higher than Longyundou 10, indicated that free proline was an important osmotic regulatory substance of drought resistance of common bean.

\section{Effect of drought stress on soluble sugar content of common bean}

Figure 5 shows that the accumulation of soluble sugar content in common bean leaves showed a single peak curve, and the peak appeared at different times according to different varieties under drought stress. The peaks of soluble sugar content of Longyundou 17 appeared on the 10th and 8th day after severe drought stress, and the peaks were $6.52 \mathrm{mg} \mathrm{g}^{-1}$ and $5.61 \mathrm{mg} \mathrm{g}^{-1}$ after mild drought stress, respectively, which were 2.2 times and 1.84 times higher than those under normal water condition. Under severe and mild drought stress, the peaks of soluble sugar content of Longyundou 10 appeared on the 8th day after stress, and the peak values were $4.63 \mathrm{mg} \mathrm{g}^{-1}$ and $3.78 \mathrm{mg} \mathrm{g}^{-1}$, respectively, which increased 1.6 times and 1.3 times as much as that under normal water condition. The soluble sugar content of Longyundou 17 was higher than that of Longyundou 10 at all stages and treatments. In the early stage of drought stress, the soluble sugar content of common bean increased significantly. With the prolongation of drought time, the accumulation of soluble sugar was seriously inhibited, especially under severe drought stress, the content of soluble sugar decreased sharply. The decrease of soluble sugar content in Longyundou 17 occurred 14 days after drought treatment with the soluble sugar content decreases by $16.9 \%$ and $22.5 \%$ under severe and mild drought stress, respectively, compared with those in normal water. Under drought stress, the content of soluble sugar in Longyundou 10 decreased earlier than that in Longyundou 17. After 10 days of severe drought stress, the content of soluble sugar in Longyundou 10 was significantly lower than that in normal water.
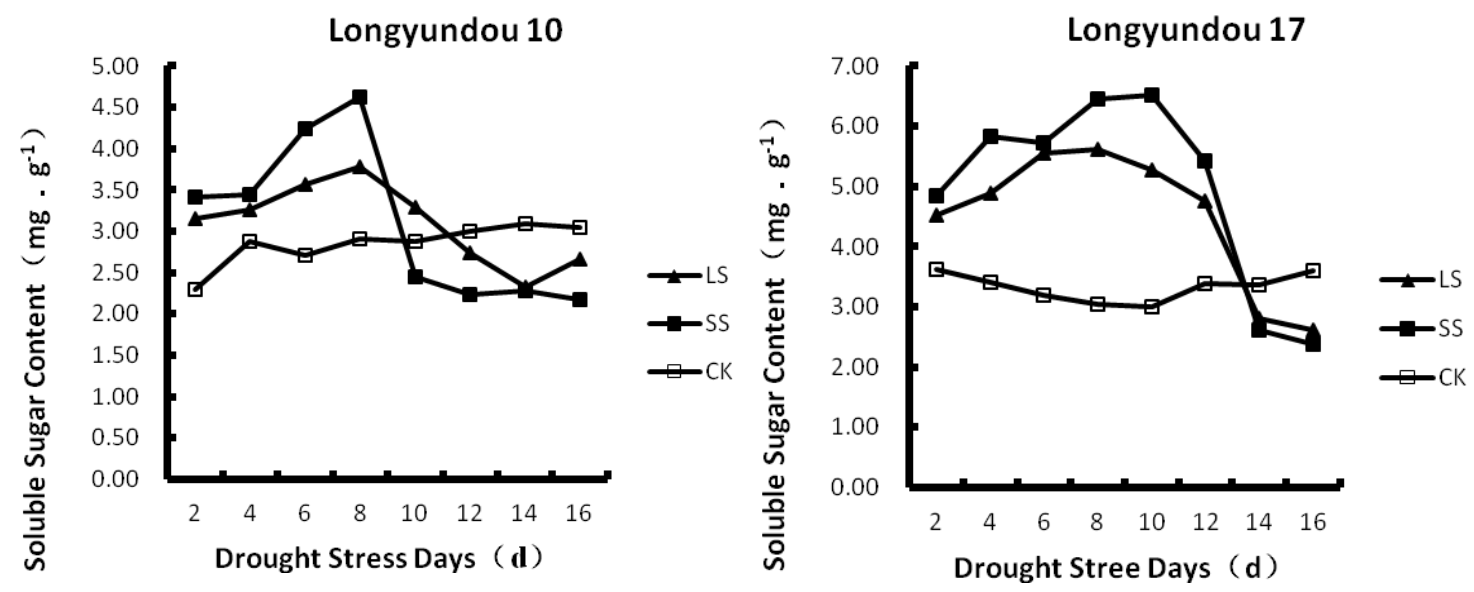

Figure 5. Changes of soluble sugar content in common bean leaves under drought stress

\section{Effect of drought stress on soluble protein content of common bean}

In Figure 6, the accumulation of soluble protein in common bean leaves at seedling stage showed a fluctuating upward trend under normal water conditions. The 
accumulation dynamics of soluble protein showed irregular changes under drought stress. At the initial stage of drought stress, soluble protein content increased, especially under severe drought stress. However, with the increase of drought stress time, the accumulation of soluble protein in common bean was inhibited, and the content was lower than that in normal water condition. Under normal water condition, the soluble protein content of Longyundou 10 was slightly higher than that of Longyundou 17.
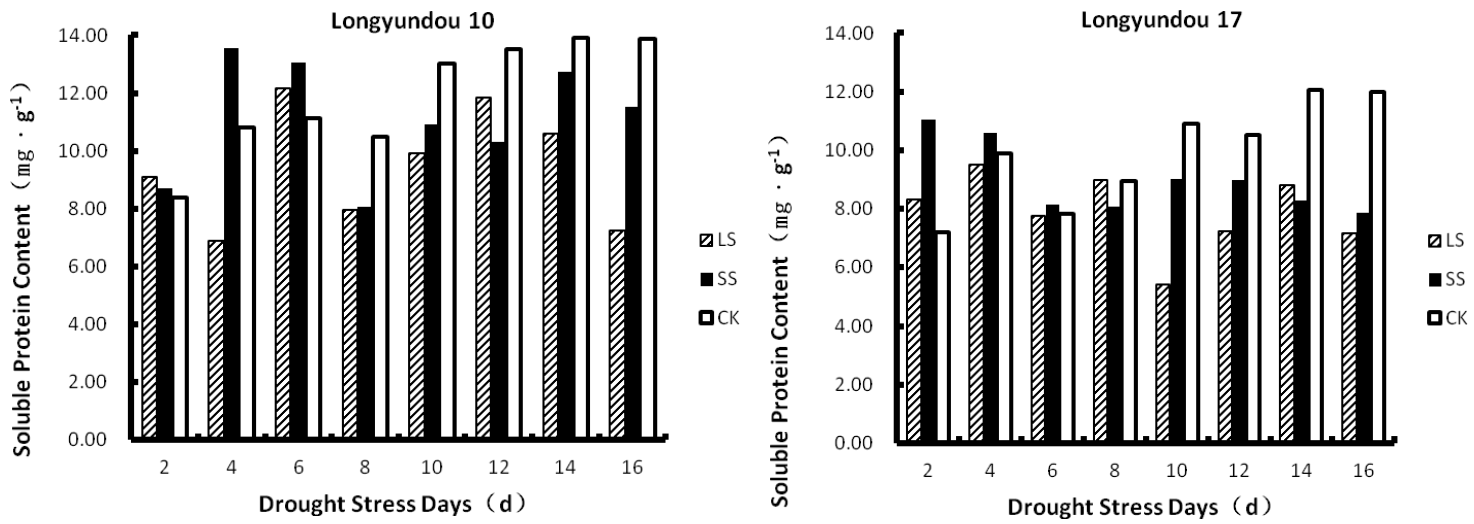

Figure 6. Changes of soluble protein content in common bean leaves under drought stress

\section{Discussion}

\section{Changes of endogenous hormones in common bean under drought stress}

Many researches have proved that plant endogenous hormones play an important regulatory role under drought stress (De Ollas et al., 2016; Sharp et al., 2004; Kurepin et al., 2015; Forner-Giner et al., 2011). Different crops and their ABA, IAA and CTK contents have different response mechanisms. Plant hormone ABA plays an important regulatory role in plant growth, development, stress resistance, stomatal movement and gene expression (Neill et al., 1999).

In the present study, ABA accumulation in common bean leaves increased significantly under mild drought stress, indicating that mild drought stress stimulated the ABA response, but severe drought stress would destroy the formation and accumulation of ABA. The ABA content in drought resistance variety Longyundou 17 was significantly higher than that in non drought-resistant variety Longyundou 10. Therefore, we believe that $\mathrm{ABA}$ can be used as important drought resistance indexes of common bean at Seedling Stage.

CTK is formed and accumulated in the root system, which is transported to the the aboveground part. At the same time, CTK is formed in large quantities in the root system under drought stress. In our study, CTK increased in the leaves of common bean seedlings under drought stress, especially in common bean varieties with stronger drought resistance. Drought stress stimulated the CTK production in Longyudou 17, suggested that moderate drought stress exercise is helpful to the stress-resistant growth of common bean.

Endogenous IAA is synthesized by plant apical tissues and growing leaves as a growth hormone. Under drought stress, the change of IAA content increases in some plants (Zhao et al., 2012; Mei et al., 2017), while decreases in others (Zhang et al., 2018; Chang et al., 2012). In the present study, IAA content in common bean leaves 
decreased in a fluctuation way under drought stress. IAA content alternate change under severe stress and mild stress. No change was observed in IAA content under two drought stresses, indicating that the mechanism of IAA response to drought stress was complex, which was consistent with previous studies.

\section{Changes of osmotic regulatory substances in common bean under drought stress}

Osmotic regulation is not only an important way for plants to resist drought adversity, but also an important physiological mechanism for plants to induce protective response under drought stress (Cabuslay et al., 2002; Alla et al., 2012; Moustakas et al., 2011). Under drought stress, osmotic regulatory substances such as proline and soluble sugar accumulate constantly in plant cells, reduce cell osmotic potential and improve water retention capacity through response, thus enhance drought resistance of crops. However, there are some differences in the accumulation of osmotic regulatory substances among different crops (Osmotic et al., 2010; Trung et al., 2018). In the present study, two common bean cultivars with obvious difference in drought resistance were chose to analyze osmotic regulatory substance changes under drought stress. These results showed that different osmotic regulatory substances had different responses. Among them, the contents of soluble sugar and proline were correlated with the drought resistance of cultivars. The drought-resistant varieties had higher expression level under drought stress compared with non drought-resistant varieties, but the soluble protein did not show the same conclusion.

\section{Conclusion}

Endogenous hormones and osmotic regulatory substances showed differences in different degrees of drought stress and among common bean varieties. When common bean was subjected to mild drought stress, the endogenous hormones and osmotic regulatory substances of common bean showed a positive response. The contents of ABA, proline and soluble sugar in drought-resistance common bean varieties were higher than those in varieties with weak drought resistance. Therefore, these substances could be considered as important indicators for drought resistance breeding.

The accumulation of endogenous hormones and osmotic regulatory substances in common bean was severely affected when drought stress lasted for a long time. Although common bean could respond to drought stress, the accumulation of related regulatory substances would be inhibited with the persistent threat of drought stress. This also indicated that the tolerance of common bean to drought stress was weak at seedling stage. Therefore, it should pay special attention to water management in seedling stage in the production of common bean.

In the present study, we discussed the response mechanism of endogenous hormones and osmotic regulatory substances of common bean under drought stress, and found the response law. We will further study the response mechanism at molecular level, especially for ABA, proline and soluble sugar, which can be used as physiological indicators of drought resistance of common bean, and explore and utilize related genes to change the physiological and biochemical characteristics of common bean for the improvement of drought-resistance of common bean. 
Acknowledgements. This research was supported by China Agriculture Research System (CARS-09); National Infrastructure for Crop Germplasm Resources (NICGR2018-024) and Protection and Utilization of Crop Germplasm Resources of Ministry of Agriculture (2130135).

\section{REFERENCES}

[1] Acharya, B. R., Assmann, S. M. (2009): Hormone interactions in stomatal function. Plant Molecular Biology 69(4): 451-462.

[2] Acostagallegos, J. A, Kelly, J. D., Gepts, P. (2007): Prebreeding in common bean and use of genetic diversity from wild germplasm. - Crop Science 47(Supplement_3): S-44-S-59.

[3] Andrés, J. C., Dominique, T., Carolina, C., Santiago, M., Matthew, W. B. (2012): Nucleotide diversity patterns at the drought-related DREB2 encoding genes in wild and cultivated common bean (Phaseolus vulgaris L.). - Theoretical \& Applied Genetics 125(5): 1069-1085.

[4] Bai, Z. Y., Li, C. D., Liu, Y. (2007): Relationship between chromosome and changing of leaf proline and protein content under drought stress in wheat (Triticum aestivum L.). Journal of Plant Genetic Resources 8(3): 325-330.

[5] Bartels, D., Sunkar, R. (2005): Drought and salt tolerance in plants. - Critical Reviews in Plant Sciences 24(1): 23-58.

[6] Basu, P. S., Berger, J. D., Turner, N. C., Chaturvedi, S. K., Ali, M., Siddique, K. H. M. (2010): Osmotic adjustment of chickpea (Cicer arietinum) is not associated with changes in carbohydrate composition or leaf gas exchange under drought. - Annals of Applied Biology 150(2): 217-225.

[7] Beebe, S. E., Rao, I. M., Cajiao, C. (2008): Selection for drought resistance in common bean also improves yield in phosphorus limited and favorable environments. - Crop Science 48(2): 582-592.

[8] Carlos, D. O., Ian, C. D. (2016): Physiological impacts of ABA-JA interactions under water-limitation. - Plant Molecular Biology 91(6): 641-650.

[9] Carolina, L., Mark, W., Juan, P. M., Daniel, V., Rodrigo, M., Erik, H. M., Claudio, P., Bartolomeo, L., Paulo, V., Peter, H., Manuel, P. (2006): Differential adaptation of two varieties of common bean to abiotic stress: I. Effects of drought on yield and photosynthesis. - Journal of Experimental Botany 57(3): 699-709.

[10] Cutler, S. R., Rodriguez, P. L., Finkelstein, R. R., Abrams, S. R. (2010): Abscisic acid: emergence of a core signaling network. - Annual Review of Plant Biology 61(1): 651679.

[11] Estela, R. A., Vinicius, N. R., Cleber, V. G. A., Alisson, F. C., Thomas, C. R. W. (2016): Biochemical indicators of drought tolerance in the common bean (Phaseolus vulgaris L.). - Euphytica 210(2): 277-289.

[12] Farooq, M., Wahid, A., Kobayashi, N., Fujita, D., Basra, S. M. A. (2009): Plant drought stress: effects, mechanisms and management. - Agronomy for Sustainable Development 29(1): 185-212.

[13] Forner-Giner, M., Ángeles, R. J., Eduardo, P., Domingo, J. I. (2011): Hydraulic and chemical responses of citrus seedlings to drought and osmotic stress. - Journal of Plant Growth Regulation 30(3): 353-366.

[14] Franca, M. G. C., Pham, T. A. T., Pimentel, C., Rossiello, R. O. P., Fodil, Y. Z., Laffray, D. (2000): Differences in growth and water relations among Phaseolus vulgaris cultivars in response to induced drought stress. - Environ Exp Bot 43: 227-237.

[15] Gloria, S. C., Osamu, I., Arcelia, A. A. (2002): Physiological evaluation of responses of flee (Oryza sativa, L.) to water deficit. - Plant Science 163(4): 815-827.

[16] He, M., Shi, D. W., Hu, Y. A., Xie, Y. F. (2007): Gender differences in the growth and endogenous hormone contents of male and female Ginkgo biloba under drought stress. Acta Agriculturae Universitatis Jiangxiensis 39(6): 1154-1162. 
[17] Kristin, A. S., Rigoberto, R., Francisco, I., Benito, C., Jorge, A. A., Porfirio, R., Nasrat, W., James, D. K. (1997): Improving common bean performance under drought stress. Crop Science 37(1): 43-50.

[18] Kuto, T., Golob, T., Kac, M. (2003): Dietary fibre content of dry and processed beans. - Food Chemistry 80(2): 231-235.

[19] Leonid, V. K., Alexander, G. I., Mohammad, Z., Richard, P. P., Suleyman, I. A., Vaughan, H., Norman, P. A. H. (2015): Stress-related hormones and glycinebetaine interplay in protection of photosynthesis under abiotic stress conditions. - Photosynthesis Research 126(2-3): 221-235.

[20] Li, L., Wang, L. F., Wu, J., Jing, R. L., Wang, S. M. (2014): Physiological characteristics of drought resistance in common bean (L.). - Acta Agronomica Sinica 40(4): 702-710.

[21] Liu, C. H., Zhou, S. S., Zhou, Y. J., Liang, D., Ma, F. W. (2012): Changes of endogenous hormones contents of two different drought-tolerant Malus rootstocks in response to drought stress. - Agricultural Research in the Arid Areas 35(5): 94-98.

[22] Mamdouh, M. N. A., Abdel-Hamid, A. K., Mamdouh, M. S., Amina, Z. A., Reham, M. N. (2012): Regulation of metabolomics in Atriplex halimus growth under salt and drought stress. - Plant Growth Regulation 67(3): 281-304.

[23] Miguel, A. R., Edilia, O., Rocío, R., Yadira, O., Jorge, A., Alejandra, A. C. (2012): Physiological analysis of common bean (Phaseolus vulgaris L.) cultivars uncovers characteristics related to terminal drought resistance. - Plant Physiology \& Biochemistry 56: 24-34.

[24] Moustakas, M., Sperdouli, I., Kouna, T., Antonopoulou, C., Therios, I. (2011): Exogenous proline induces soluble sugar accumulation and alleviates drought stress effects on photosystem II functioning of Arabidopsis thaliana leaves. - Plant Growth Regulation 65(2): 315-325.

[25] Neill, S. J., Burnett, E. C. (1999): Regulation of gene expression during water deficit stress. - Plant Growth Regulation 29(1-2): 23-33.

[26] Robert, E. S., Valeriy, P., Lindsey, G. H., William, G. S., Gordon, K. S., Hans, J. B., Henry, T. N. (2004): Root growth maintenance during water deficits: physiology to functional genomics. - Journal of Experimental Botany 55(407): 2343-2351.

[27] Sauter, A., Davies, W. J., Hartung, W. (1997): The long-distance abscisic acid signal in the droughted plant: the fate of the hormone on its way from root to shoot. - Journal of Experimental Botany 52(363): 1991-1997.

[28] Schindler, U., Steidl, J., Mailer, L. (2007): Drought risk to agricultural land in Northeast and central Germany. - Joumal of Plant Nutrition \& Soil Science 170(3): 357-362.

[29] Schroeder, J. I., Kwak, J. M., Allen, G. J. (2001): Guard cell abscisic acid signalling and engineering drought hardiness in plants. - Nature 410(6826): 327-30.

[30] Tang, L. S., Li, Y., Zhang, J. H. (2005): Physiological and yield responses of cotton under partial rootzone irrigation. - Field Crops Research 94(2-3): 214-223.

[31] Trung, D. T., Li, J., Zhang, F. J., Xing, Y. X., Yang, L. T., Li, Y. R., Thi, H. N. (2018): Changes of antioxidant enzyme activities and contents of osmotic regulation substances in leaves of different sugarcane varieties under drought stress. - Chinese Journal of Tropical Crops 39(5): 858-866.

[32] Verslues, P. E., Zhu, J. K. (2005): Before and beyond ABA: upstream sensing and internal signals that determine ABA accumulation and response under abiotic stress. Biochemical Society Transactions 33(2): 375-379.

[33] Yang, Y. B., Zhu, Y. Y., Gao, Y., Ren, G. X. (2016): Comparisons of phaseolin type and $\alpha$-amylase inhibitor in common bean (Phaseolus vulgaris L.) in China. - Crop Journal 4(1): 68-72.

[34] Yordanov, I., Velikova, V., Tsonev, T. (2000): Plant responses to drought, acclimation, and stress tolerance. - Photosynthetica 38(2): 171-186. 


$$
-4457 \text { - }
$$

[35] Zhang, H. Y., Duan, W. X., Xie, B. T., Dong, S. X., Wang, B. Q., Shi, C. Y., Zhang, L. M. (2018): Effects of drought stress at different growth stages on endogenous hormones and its relationship with storage root yield in sweet potato. - Acta Agronomica Sinica 44(1): 126-136.

[36] Zhao, M. R., Han, Y. Y., Feng, Y. N., Li, F., Wang, W. (2012): Expansions are involved in cell growth mediated by abscisic acid and indole-3-acetic acid under drought stress in wheat. - Plant Cell Reports 31(4): 671-685. 